\title{
PARAMETRIC STUDY OF THE DEVICE ANGLE DEPENDENCY OF A SINGLE VORTEX GENERATOR ON A FLAT PLATE.
}

U. Fernández ${ }^{1}$ (unai.fernandez@ehu.es), P.-E. Réthoré ${ }^{2}$ (pire@dtu.dk), N. N. Sørensen ${ }^{2}$ (nsqr@dtu.dk), Clara M. Velte ${ }^{3}$ (cmve@dtu.dk), F. Zahle ${ }^{2}$ (frza@dtu.dk) and E. Egusquiza ${ }^{4}$ (egusquiza@mf.upc.edu).

${ }^{1}$ University of the Basque Country, Nuclear Engineering and Fluid Mechanics Department, Nieves Cano 12, 01006 Vitoria-Gasteiz, Araba, Spain.

${ }^{2}$ Aeroelastic Design Program, Department of Wind Energy, Technical University of Denmark, Frederiksborgvej 399, DK-4000 Roskilde, Denmark.

${ }^{3}$ Department of Wind Energy, Technical University of Denmark, Nils Koppels Allé, 2800 Kgs. Lyngby, Denmark.

${ }^{4}$ Technical University of Catalonia, Fluid Mechanics Department, Av. Diagonal, 64708028 Barcelona Spain.

Abstract. A detailed study of the device angle dependency of a single vortex generator $(V G)$ is presented in this paper. A single Vortex Generator on a test section wall case, with four different positions of the device angle to the incoming flow, has been designed and solved by computational methods. The computational fluid dynamic (CFD) simulations have been compared with a wind tunnel experiment, where the corresponding parametric study was performed over a single vane mounted on the test section wall in low-speed wind tunnel. In this experiment the flow was recorded using Stereoscopic Particle Image Velocimetry (S-PIV) in cross-planes at various positions downstream of the vane.

The main goal of this article is to study the angle dependency of a single VG mounted on a test section wall; for this purpose CFD simulations have been carried out and compared with a wind tunnel experiment and an analytical model.

Keywords: Vortex Generators, CFD, computational fluid dynamic, parametric study.

\section{INTRODUCTION}

Vortex generators (VG) are commonly used devices to control boundary layer separation. These devices are usually a triangular or rectangular vanes inclined at an angle to the incoming flow to generate a vortex shedding. These generators are usually dimensioned in relation to the local boundary layer height to allow for the best interaction between the vortex and boundary layer, and are usually placed in groups of two or more upstream of the flow separation area, Anderson [1].

Vortex Generators have been investigated for more than fifty years in applied aerodynamics on airplane wings, Taylor [2, 3, 4] and Wentz [5]. These passive devices are used for flow control, modifying the boundary layer motion and bringing high momentum fluid down 
into the near wall region of the boundary layer, Figure 1. Through this transfer of energy, the velocity of the inner region is increased at the same time as the boundary layer thickness is decreased, which in turn causes the separation of the flow to be delayed, Rao et al. [6].

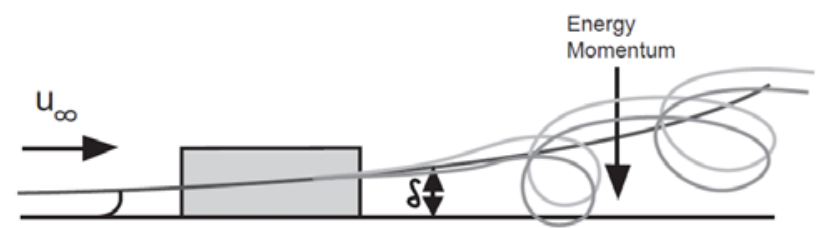

Figure 1: Boundary layer motion alteration by a rectangular VG.

Basic research on Vortex Generators mounted on a flat plate has previously occupied several researchers, Lin et al. [7]. Later investigations at moderate Reynolds number made by Kerho et al. [8] with vortex venerators used to control laminar separation bubbles, showed a significant drag reduction. Also Lin et al. [9] saw the Drag reducing and the Lift increasing effect of sub boundary layer VG. Wendt et al. [10] investigated an array of VG experimentally where the VGs were aligned to generate counter rotating vortices.

Vortex Generators are applied on wind turbine blades with the aim to delay or prevent the separation of the flow and to decrease roughness sensitivity of the blade. They are usually mounted in a spanwise array on the suction side of the blade and have the advantage that they can be added as a post-production fix to blades that do not perform as expected. Vortex Generators extend the lift curve by suppressing turbulent separation through mixing of the outer flow of the airfoil with the boundary layer. The delay of turbulence separation leads to higher maximum lift values and increased stall angles. An overview of different airfoils with several VG options is listed in van Rooij and Timmer [11]. So, adding VGs in wind turbine blades is a simple solution to improving the performance of a rotor, Schubauer et al. [12] and Bragg et al. [13].

The effect of VGs in a 1MW Wind Turbine was investigated by Øye [14], where a comparison between the measured power curve on a wind turbine with and without VGs, showed that VGs can be applied successfully, increasing the output power for nearly all wind speeds.

In the present study CFD simulations have been carried out by EllipSys3D CFD Code, Michelsen [15] and Sørensen [16], and compared with a wind tunnel experiment, where a parametric study were performed over a single vane mounted on the test section wall in lowspeed wind tunnel. In this experiment the flow was recorded using Stereoscopic Particle Image Velocimetry in cross-planes at various positions downstream of the vane. The experimental conditions and setup are the same as described in Velte et al. [17]. In order to qualitatively compare the model with the measurement, the analytical VG model performed in the thesis work by Velte [18] is used.

The main objective of this article is to study the angle dependency of a single VG mounted on a flat plate; for this purpose CFD simulations have been carried out and compared with a wind tunnel experiment and an analytical VG model. So, the proposal of this research has been divided as Figure 2 shows. 


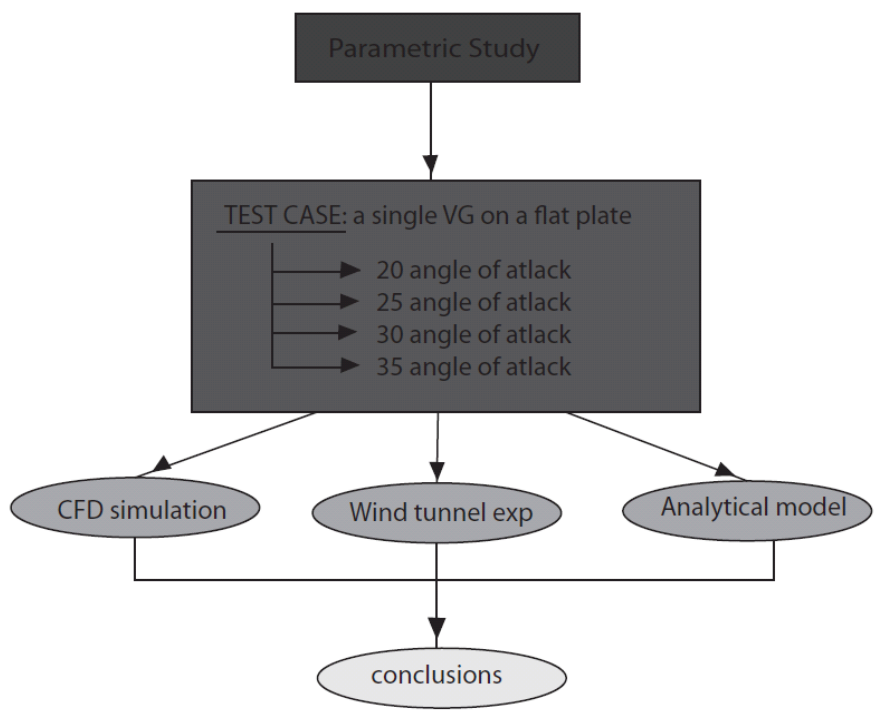

Figure 2: article lay-out

In all cases (CFD simulations, wind tunnel experiments and analytical model) the measurements have been conducted in a spanwise plane, normal to the test section floor, positioned five VG heights downstream of the vortex generator trailing edge when the angle of the device is zero, see Figure 3.

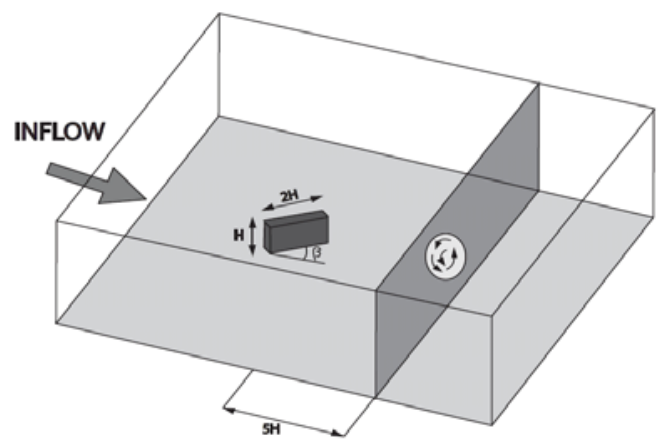

Figure 3: Plane location where the measurements were conducted.

\section{COMPUTATIONAL CONFIGURATION.}

In the present work, steady state computations have been carried out and are compared to the experimental observations. These CFD computations were performed using the EllipSys3D code, Michelsen [15] and Sørensen [16], as described in [19]. This in house CFD code is a structured finite-volume flow solver using, in this work, Reynolds-Averaged NavierStokes equations. The pressure/velocity copling is ensured using the SIMPLE algoritm and only steady-state computations have been performed. The convective terms are discretized utilising the third order Quadratic Upstream Interpolation for Convective Kinematics (QUICK), Khosla et al. [22]. For these computations the $k-\omega S S T$ (Shear Stress Transport) turbulence model by Menter [20] was used.

This case consists in a single VG on a flat plate and the computational domain has been defined with the following dimensions, normalized with the VG height, Figure 4. The flow domain width is 32 times the VG height and the height is 10 times. The flow domain length is 30 times the VG height in order to capture the generated vortex. 


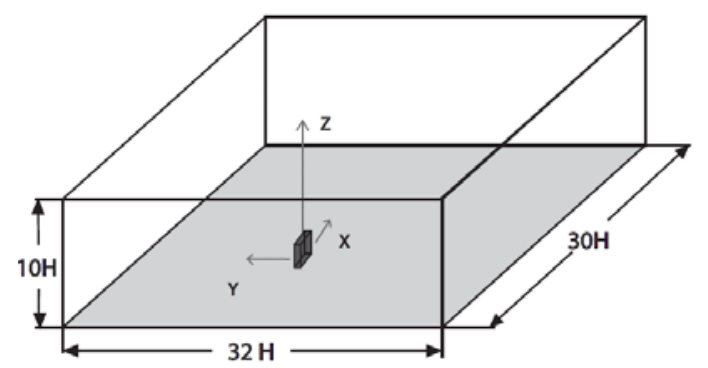

Figure 4: Computational domain of the CFD case.

The geometry dimensions of the rectangular VG are defined with a length of two times the VG height; see Figure 5(a). The thickness of the vane is constant and with no sharp edges. A boundary layer is developed over test section floor, forced by the viscous interaction between the wall and the flow. The VG was positioned test section floor in such way that the boundary layer thickness at this location is equal to the VG height.

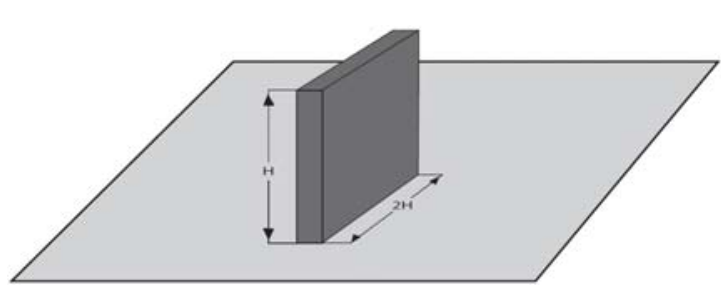

(a) VG dimensions

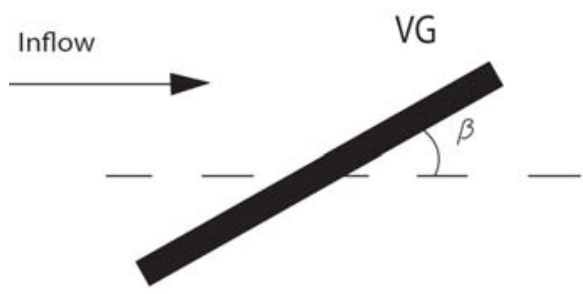

(b) VG Lay-out

Figure 5: VG geometry.

The angle of attack defined is $\beta$ degrees and four different angles have been selected for the parametric study: $\beta=20^{\circ}, 25^{\circ}, 30^{\circ}$ and $35^{\circ}$, Figure $5(\mathrm{~b})$.

The Reynolds Number based on VG height $H$ is: $\quad \operatorname{Re}=\frac{\rho U_{\infty} H}{\mu}=2500$

where $\rho$ is the density, $\mu$ the viscosity and $U_{\infty}$ the free stream velocity. The computational setup of the CFD simulations consists in a block structured mesh of 18 million cells with the first cell height $(\Delta \mathrm{z} / \mathrm{H})$ of $1.510^{-6}$ dimensionalized by the VG height. Around the VG geometry, the mesh has $510^{6}$ cells, while the mesh downstream the VG for capturing the wake has aproximatly $2.510^{6}$ cells, Figure 6 . In order to resolve the boundary layer, cell clustering has been used close to the wall and the dimensionless distance from the wall is less than $2(y+<2)$, as the SST turbulence model requires.

Verification of the mesh was performed by a mesh dependency study. Results obtained for the finer mesh ( 66 blocks of $65^{3}$ cells) are compared with results obtained for a standard ( 66 blocks of $33^{3}$ cells) and a coarser mesh ( 66 blocks of $17^{3}$ cells). Figure 16 of Appendix $\mathrm{C}$ illustrates the axial and azimuthal velocities profiles for all device angles of attack. A mesh dependency of around 5\% has been detected. 


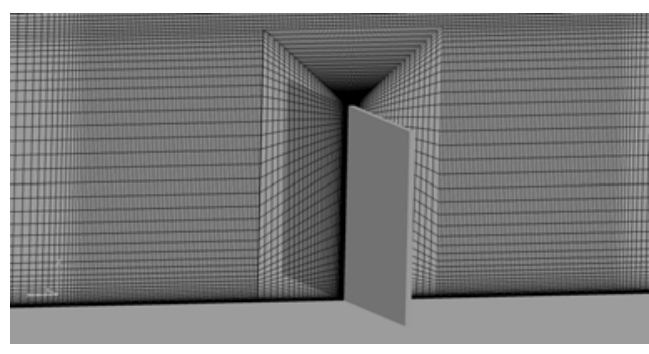

(a) Cross flow section

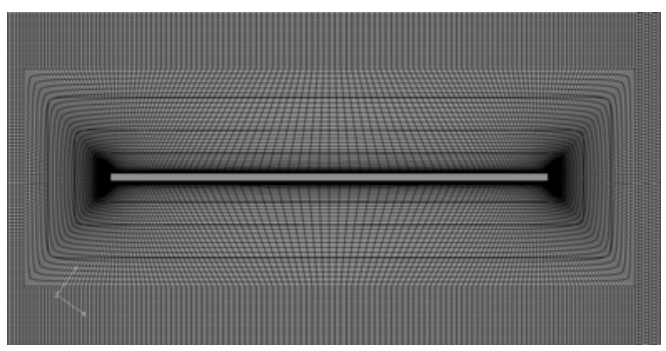

(b) Horizontal section

Figure 6: Mesh Sections on the VG.

\section{WIND TUNNEL EXPERIMENTS DESCRIPTION.}

Considering the test section setup in Figure 7, the measurements were carried out in a closed-circuit wind tunnel with an 8:1 contraction ratio and a test section of cross-sectional area $300 \times 600 \mathrm{~mm}$ with length $2 \mathrm{~m}$. At the inlet of the test section, a turbulence-generating grid with mesh length $39 \mathrm{~mm}$ was situated.

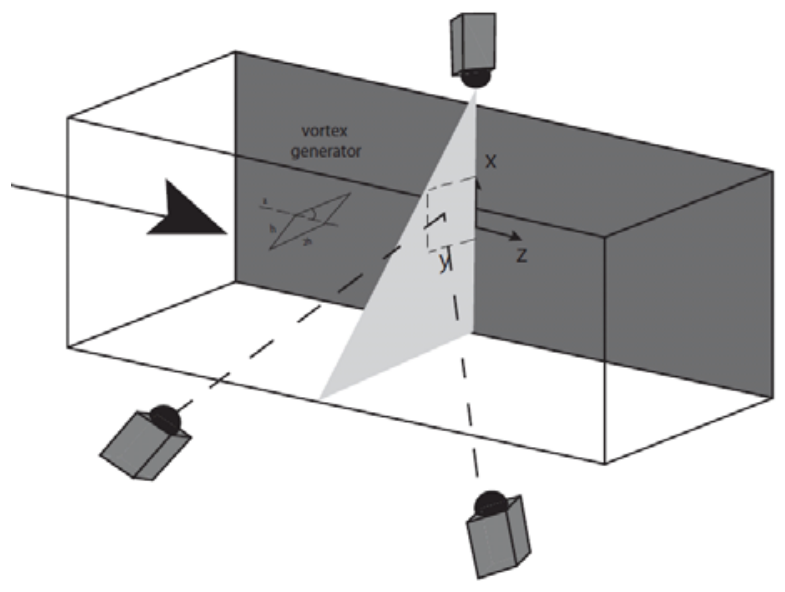

Figure 7: Schematic illustration of the experimental set-up and device geometry. The large arrow to the left indicates the main flow direction and $\beta$ the device angle.

The experiments were conducted with a variable angle $\beta$ to the incoming flow in a low-Reynold-number flow ( $\mathrm{Re}=2600$ based on an inlet turbulence-generating grid size $\mathrm{L}=39 \mathrm{~mm}$ and free stream velocity $U_{\infty}=1.0 \mathrm{~m} \mathrm{~s}^{-1}$ ). The wind tunnel speed was obtained by measuring the pressure drop across an orifice plate. The turbulence intensity at the inlet from laser doppler anemometry (LDA) measurements has been found to be $13 \%$. The boundary layer thickness at the position of the vortex generator has been estimated from LDA measurements to be approximately $\delta_{V G}=25 \mathrm{~mm}$. The actuator is a rectangular vane of the same height as the local boundary layer thickness, $h=\delta_{V G}$, with a length of $2 h$. The vortex generator was positioned on a vertical wall in the center of the test section with its trailing edge 750 $\mathrm{mm}$ downstream of the inlet grid when it is at zero angle to the mean flow.

The measurements were conducted in a spanwise plane, with plane normal parallel to the test section walls, positioned five device heights downstream of the vortex generator. The SPIV equipment was mounted on a rigid stand and included a double cavity New Wave Solo 120XT Nd-YAG laser (wavelength $532 \mathrm{~nm}$ ) capable of delivering light pulses of $120 \mathrm{~mJ}$. The 
pulse width, i.e., the duration of each illumination pulse, was $10 \mathrm{~ns}$. The light-sheet thickness at the measurement position was $2 \mathrm{~mm}$ and was created using a combination of a spherical convex and a cylindrical concave lens. The equipment also included two Dantec Dynamics Hi Sense MkII cameras $(1344 \times 1024$ pixels $)$ equipped with $60 \mathrm{~mm}$ lenses and filters designed to only pass light with wavelengths close to that of the laser light.

\section{ANALYTICAL MODEL FEATURES.}

The starting point is the classical Lamb-Oseen vortex model; see Figures 8 (a),

$$
\omega_{r}=0 ; \quad \omega_{\theta}=0 ; \quad \omega_{z}=\frac{\Gamma}{\pi \varepsilon^{2}} \exp \left(-\frac{r^{2}}{\varepsilon^{2}}\right)
$$

where $\omega_{r}, \omega_{\theta}$ and $\omega_{z}$ are radial, rotational and axial vorticity, respectively. $\Gamma$ is the vortex core circulation, $\varepsilon$ the vortex core radius and $r$ the radial coordinate in the cylindrical coordinate system. This model merely includes rotation in the plane perpendicular to the axis of the longitudinal vortex. From measurements and computational results however, one can clearly observe an induced velocity in the axial direction, see Figure 8 (b). Similarly to an electrical coil inducing a magnetic field when passing current through the wires, the vorticity lines can induce a velocity field. With the helical shape of the vorticity lines, Figure 8(b), having a nonzero value of $\omega_{\theta}$, the axial velocity deficit can be explained.
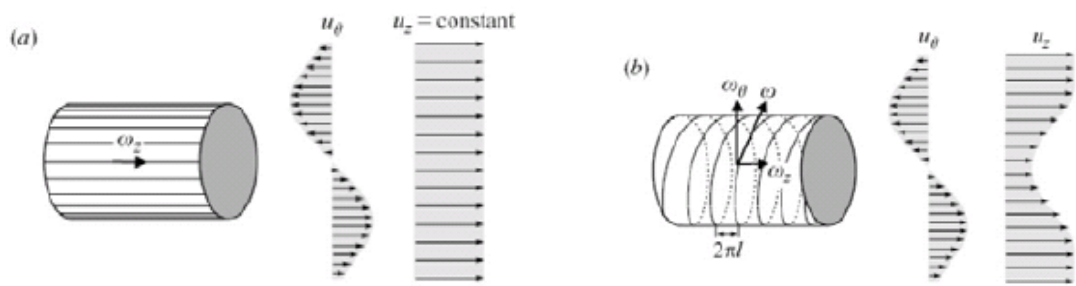

Figure 8: Sketch of vorticity field and induced velocity profile by Lamb-Oseen vortex with rectilinear vortex lines (a) and Batchelor vortex with helical structure of vortex lines (b).

$$
\omega_{r}=0 ; \quad \omega_{\theta}=r \omega_{z} / l ; \quad \omega_{z}=\frac{\Gamma}{\pi \varepsilon^{2}} \exp \left(-\frac{r^{2}}{\varepsilon^{2}}\right)
$$

$l$ is the helical pitch, i.e., the period of the helical vorticity lines. This condition for helical symmetry can also be formulated in a simple linear manner for the corresponding velocities:

$$
u_{z}=u_{0}-\frac{r}{l} u_{\theta}
$$

where the integration constant $u_{0}$ is the vortex convection velocity. $u_{0}, u z$ and $u_{\theta}$ can be found directly from the data, the circulation $\Gamma$ can be found by integrating across the vortex core and $\varepsilon$ from fitting the expression for $\omega_{z}$ to the data. The helical pitch $l$ can be found in many ways, e.g., from least square fitting of the velocity formulation above to the data. It has been observed in [17], which also describes the theory more in detail, that the Batchelor vortex model combined with the above model for helical symmetry can be applied. 


$$
u_{\theta}=\frac{\Gamma}{2 \pi r}\left[1-\exp \left(-\frac{r^{2}}{\varepsilon^{2}}\right)\right] ; \quad u_{z}=u_{0}-\frac{\Gamma}{2 \pi l}\left[1-\exp \left(-\frac{r^{2}}{\varepsilon^{2}}\right)\right]
$$

Note that this model is very general in the sense that it does not leave any restrictions on the shape of the vortex core. Further, this model can describe the full flow in a plane using merely four parameters: $\Gamma, u_{0}, l$ and $\varepsilon$. The full vortex can be described by extending this model with simple self-similarity analysis of both the wake and the rotational velocities [21].

\section{RESULTS.}

Four different angles of attack $\beta\left(20^{\circ}, 25^{\circ}, 30^{\circ}\right.$ and $\left.35^{\circ}\right)$ of the $\mathrm{VG}$ to the incoming flow were chosen for the computations and subsequently compared with the wind tunnel experiments and an analytical model as described in [18] as a quantitative comparison.

\subsection{Computational results}

CFD results of a single VG on a flat plate were performed using the EllipSys CFD code. Figures 9 (a), (b), (c) and (d) show the evolution of the vortex generated downstream the trailing edge of the VG for the CFD case with the device angle of incidence $\beta$ as a $20^{\circ}$, $25^{\circ}, 30^{\circ}$ and $35^{\circ}$, respectively. The inner separation between planes is four times the VG height.

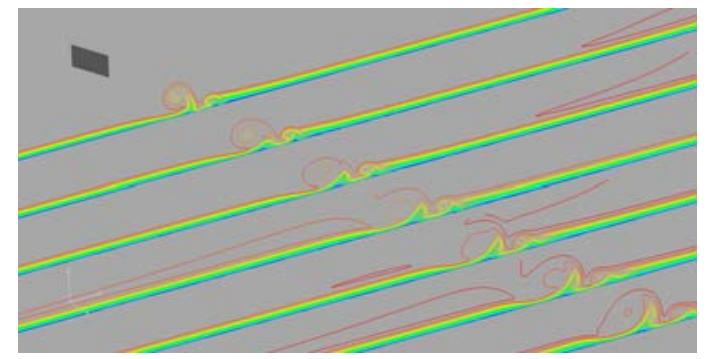

(a) $\beta=20^{\circ}$

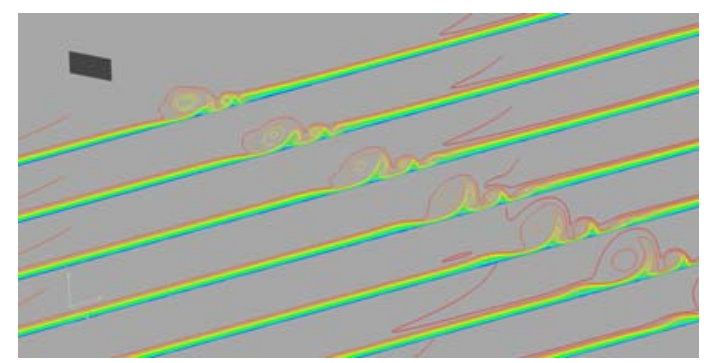

(c) $\beta=30^{\circ}$

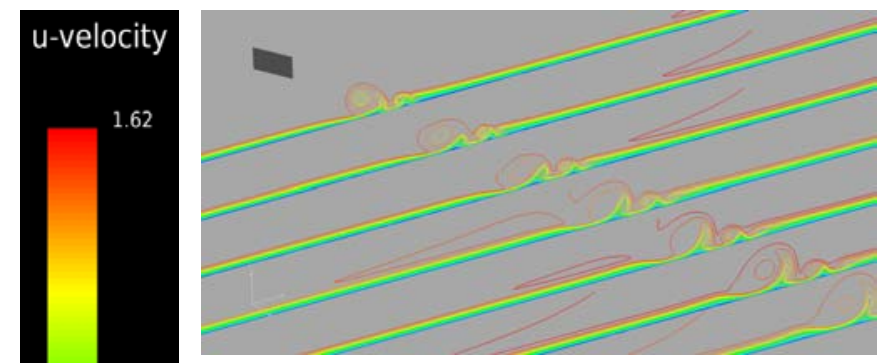

(b) $\beta=25^{\circ}$

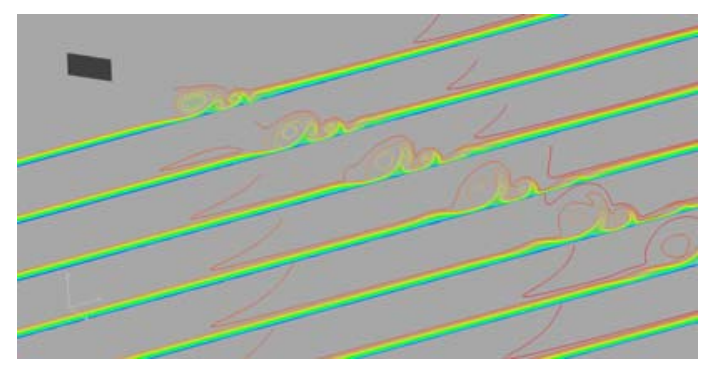

(d) $\beta=35^{\circ}$

Figure 9: CFD results of vortex development downstream the VG with four different angles of attack.

Three parameters have been chosen for a qualitative comparison between the four different angles of $\beta$ : axial velocity, vorticity and static pressure. Figures 12 and 13 (Appendix 
A) illustrate the axial velocity and vorticity fields, respectively, and Figure 14 (Appendix B) the pressure fields five device heights downstream the VG. In addition to this, a top view pressure field has been represented for each VG angle; see Figure 15 (Appendix B).

\subsection{Quantitative Comparison.}

As a quantitative comparison, wind tunnel measurements and an analytical model of the primary vortex is considered in the context of the CFD simulations. This Analytical Model described in [18], reduces the complex flow to four parameters (circulation, convection velocity, vortex core radius and helical pitch) and enables quantitative comparison in addition to the qualitative one.

Figure 10 represents the axial $u_{z}$ (upper) and azimuthal $u_{\theta}$ (lower) velocity profiles calculated for $20^{\circ}, 25^{\circ}, 30^{\circ}$ and $35^{\circ}$ degrees of the device angle. These values were extracted in a plane normal to the section wall five device heights downstream of the VG, along a line parallel to the wall passing through the centre of the primary vortex.
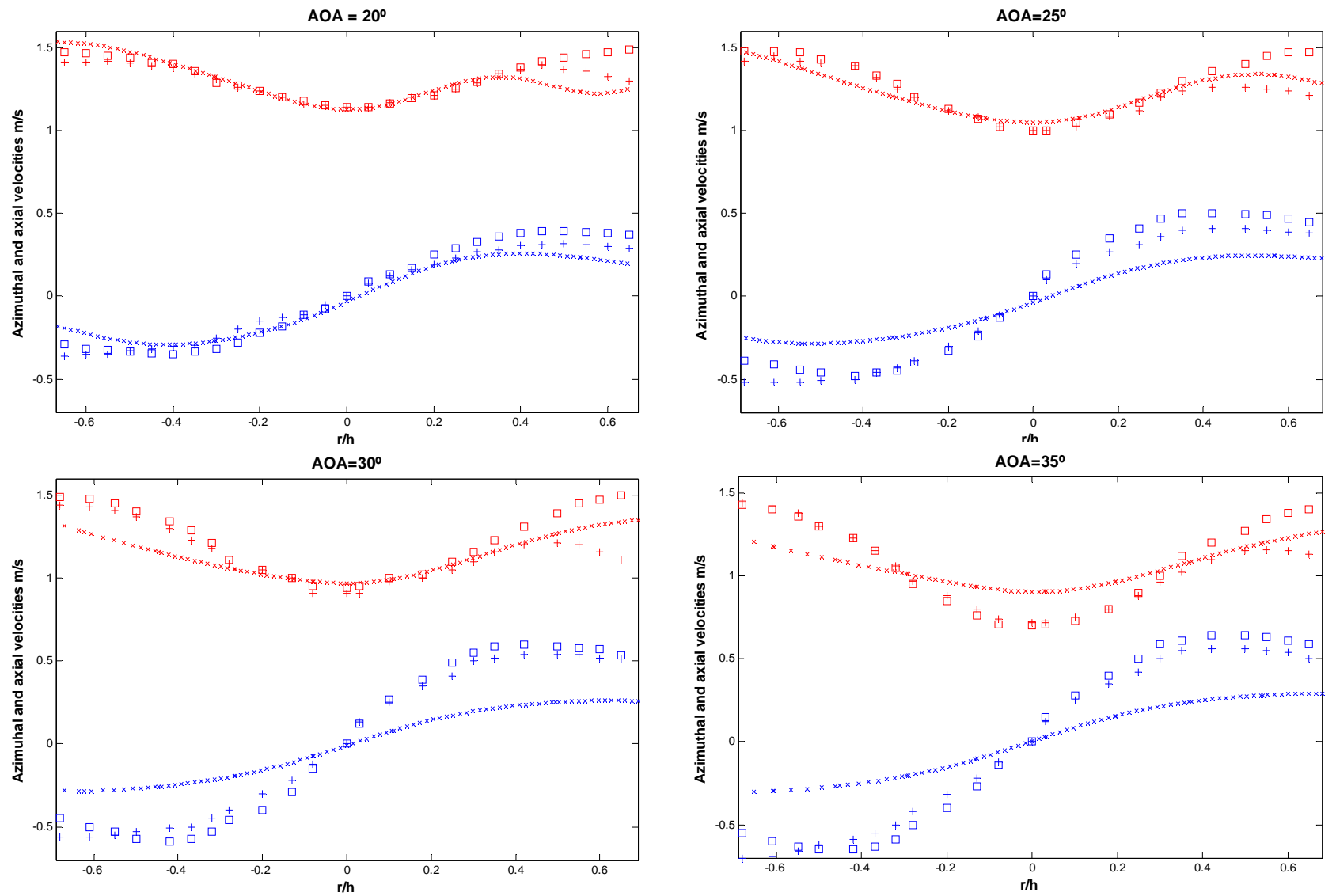

Figure 10 Comparison of axial and azimuthal velocities of embedded vortices generated by a vortex generator for four device angles $\beta$. (x) CFD results, $(+)$ Wind tunnel experimental data, $(\square)$ Analytical Model. Upper values (red colour) are the axial velocity profile $u_{z}$ and lower (Blue colour) the azimuthal velocity profile $u_{\theta}$. 
In order to analyse the quantitative differences between the computational results and the experimental data, the Root Mean Square Error RMSE has been calculated:

$$
R M S E=\sqrt{\frac{\sum_{i=1}^{n}\left(a_{1, i}-a_{2, i}\right)^{2}}{n}}
$$

The differences between the wind tunnel measurements and the CFD computations are represented in Figure 11. Green colour bars illustrate the axial velocity differences and the yellow ones the azimuthal differences, both of them between the wind tunnel experimental data and numerical results of the CFD simulations.

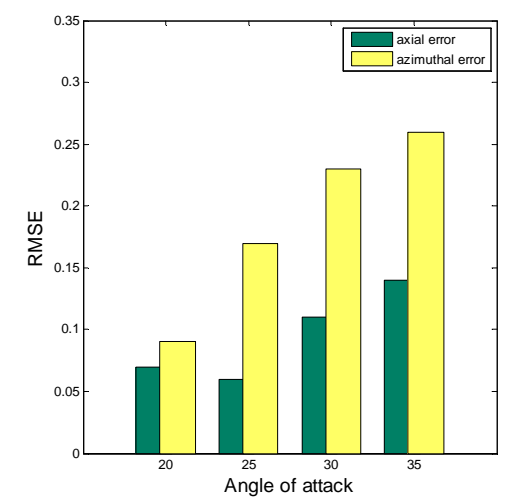

Figure 11: Root mean square error between the wind tunnel experimental data and CFD computations. Green colour bars represent the error in the axial velocity profiles and the yellow ones the azimuthal velocity profiles errors.

\section{DISCUSSION.}

Simulations results of $20^{\circ}$ and $25^{\circ}$ degrees of the device angle compare well with the experimental and the analytical model. However, significant differences were observed as the angle of attack increases. One reason for these differences could be that the boundary layer evolution along the wall was not accurately performed in the simulations to reproduce the same boundary layer profile facing the leading edge of the VG as in the wind tunnel experiments.

As Figure 10 shows, at 20 degrees of angle of attack the CFD results are well matched with wind tunnel experimental data and the analytical model, both for the axial and azimuthal velocity profiles. Also, the advection velocity in both cases matches very well. However, the perturbation from a secondary vortex (see also Figures 12-14), which is observed in the asymmetry to the right in the axial velocity profiles, seems to be stronger in the CFD case. This secondary vortex is present with variable strength at all considered device angles, introducing a disturbance in the flow field of the main vortex. However, the influence is more notable in the case of 20 degrees. In the 25 degrees of angle of attack case, the axial velocity profiles show excellent agreement in all the cases, though the azimuthal velocity profile of the CFD case starts displaying relevant differences with the wind tunnel data and the model. For 
all four VG angles it is seen that the axial velocity is predicted much better than the azimuthal one. In the azimuthal profiles, it is evident that the swirl increases with increased angle of attack. Unfortunately, the CFD simulations are not able to accurately capture this increase.The bar chart of Figure 11 illustrates that, when the device angle is increasing, the RMSE increases as well, above of all in the azimuthal velocity profiles. These differences in the azimuthal velocity profiles could be explained due to the difficulties of the turbulent models to capture the swirling flows with high accuracy.

\section{CONCLUSIONS.}

In this paper, vortices generated by a passive rectangular vane-type vortex generator of the same height as the boundary layer thickness in a flat plate have been studied. CFD computational simulations with four different angles of attack $\beta\left(20^{\circ}, 25^{\circ}, 30^{\circ}\right.$ and $\left.35^{\circ}\right)$ of the $\mathrm{VG}$ to the incoming flow have been carried out using the RANS method and compared with wind tunnel experimental data and an analytical model.

Some differences have been noticed between the computational results and the experimental ones, above of all in the azimuthal profiles. Also it was observed that the differences in the axial and azimuthal velocity profiles are more relevant as the device angle increases. More work is therefore required in order to address these problems.

For future investigations, it would be highly interesting to achieve more experiments and CFD simulations at height Reynolds numbers, which provide more realistic flow conditions for most applications. Actually, experiments have already been performed in a high Reynolds number boundary layer and are under processing. This boundary layer, acquired in a unique wind tunnel in Lille, France, can accurately follow the well known log-law etc. and these measurements will therefore naturally form a better basis for CFD validation.

\section{Acknowledgements}

The author is really grateful to the researchers of DTU Wind (Technical University of Denmark) who worked on this article and also to the Centre of Industrial Diagnosis and Fluid Dynamics CDIF at the Technical University of Catalonia. C.M. Velte and N.N. Sørensen were supported by EUDP-2009-II-Grant Journal No. 64009-0279, which is gratefully acknowledged. 


\section{REFERENCES.}

[1] Anderson B. H., "The aerodynamic characteristics of vortex ingestion for the fla-18 inlet duct", NASA Lewis Research Center, Cleveland, Ohio 44135. $29^{\text {th }}$ Aeroespace Sciences Meeting, Nevada, January 1991.

[2] Taylor, H. D. "The elimination of diffuser separation by vortex generators". Research Department Report No. R-4012-3. United Aircraft Corporation, East Hartford, Connecticut, 1947.

[3] Taylor, H. D. "Application of vortex generator mixing principles to diffusers". Research Department Concluding Report No. R-15064-5. United Aircraft Corporation, East Hartford, Connecticut, 1948.

[4] Taylor, H. D. "Summary report on vortex generators". Research Department Report No. R-05280-9. United Aircraft Corporation, East Hartford, Connecticut, 1950.

[5] Wentz W.H. jr, "Effectiveness of spoilers on the GA(W)-1 airfoil with a high performance Fowler flap" NASA CR-2538 May 1975.

[6] Rao D. M., Kariya TT. „Boundary-layer submerged vortex generators for separation control-an exploratory study". AIAA Paper 88-3546-CP, AIAA/ASME/SIAM/APS $1^{\text {st }}$ National Fluid Dynamics Congress, Cincinnati, OH, July 25-28, 1988.

[7] Lin, J.C. \& Howard, F.G. "Turbulent flow separation control through passive techniques". AIAA 2nd Shear Flow Conference, March 13- 16 1989, Tempe AZ, AIAA Paper 89-0976.

[8] Kerho M., S. Huctcherson, R. F. Blackwelder and R. H. Liebeck. "Vortex Generators used to control laminar separation bubbles". Journal of Aircraft, 30(3) 315-319, 1993.

[9] Lin, S. K. Robinson and R. J. McGhee. "Separation Control on high-lift airfoils via micro vortex generators". Journal of aircraft, 27(5):503-507, 1994.

[10] Wendt, B. J. "Parametric study of vortices shed from airfoil vortex generators". AIAA Journal 42,2185_2195, 2004.

[11] van Rooij, R. P. J. O. M. \& Timmer, W. A. "Roughness Sensitivity Considerations for Thick Rotor Blade Airfoils". AIAA-paper 2003-0350.

[12] Schubauer G. B., Spangenber W. G. "Forced mixing in boundary layers". J Fluid Mech, 1 1960;8:10-32. 
[13] Bragg M. B. Gregorek G.M. "Experimental study of airfoil performance with vortex generators". Journal of aircraft; 24(5):305-9, 1987.

[14] Øye, S. "The Effect of Vortex Generators on the Performance of the ELKRAFT 1000 kW Turbine". 9th IEA Symposium on Aerodynamics of Wind Turbines, Stockholm, Sweden, ISSN 0590_8809, 1995.

[15] Michelsen J.A. "Basis3d- a platform for development of multiblock pde solvers". Technical Report AFM 94-06, Technical University of Denmark, Dept. of Mechanical Engineering, 1994.

[16] Sørensen N. N. "General purpose flow solver applied to flow over hills". Technical Report Risoe-R-827(EN), Risoe National Laboratory, 1995.

[17] Velte, C.M., Hansen, M. O. L., Okulov, V. L., 'Helical structure of longitudinal vortices embedded in turbulent wall-bounded flow", Journal of Fluid Mechanics., 619, 167 - 177, 2009.

[18] Velte C. M. "Characterization of Vortex Generator Induced Flow”. PhD Thesis, Technical University of Denmark, Lyngby, Denmark 2009.

[19] Fernández, U., Réthoté, P.-E., Sørensen, N.N., Velte, C.M., Zahle, F., Egusquiza, E., "Comparison of Four Different Models of Vortex Generators", Proceedings of EWEA 2012.

[20] Menter F.R.., "Zonal Two equation k- $\omega$ Turbulence Model for aerodynamic flows", AIAA Journal 932906, 1993.

[21] Velte, C.M.,"A vortex generator flow model based on self-similarity", AIAA Journal, accepted for publication.

[22] Khosla P.K. and Rubin S.G. "A diagonally dominant second-order accurate implicit scheme", Computer Fluids, 207-209, 1974. 


\section{APPENDIX A}

\section{AXIAL VELOCITY AND VORTICITY FIELDS}

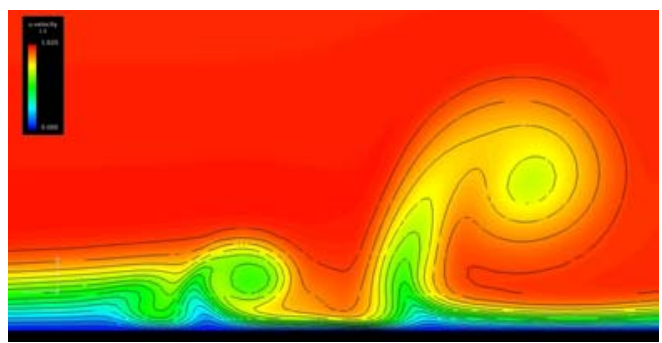

(a) $\beta=20^{\circ}$

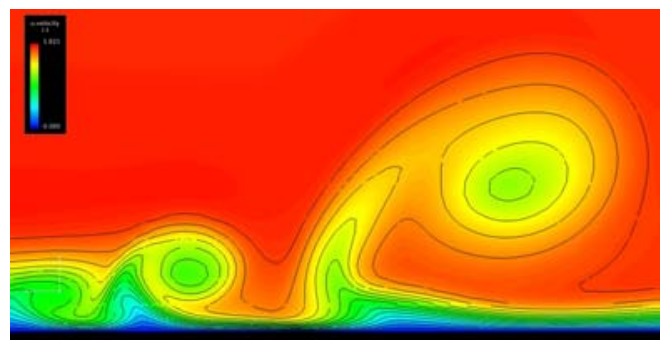

(b) $\beta=25^{\circ}$

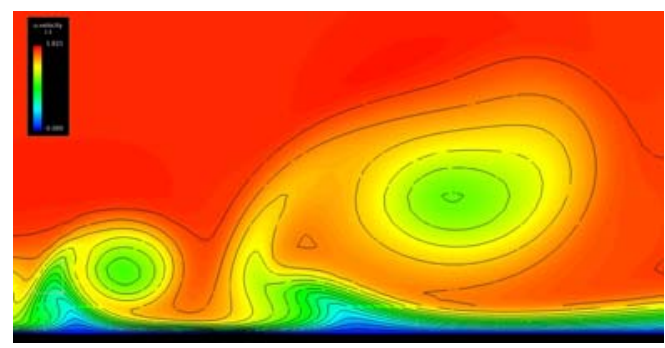

(c) $\beta=30^{\circ}$

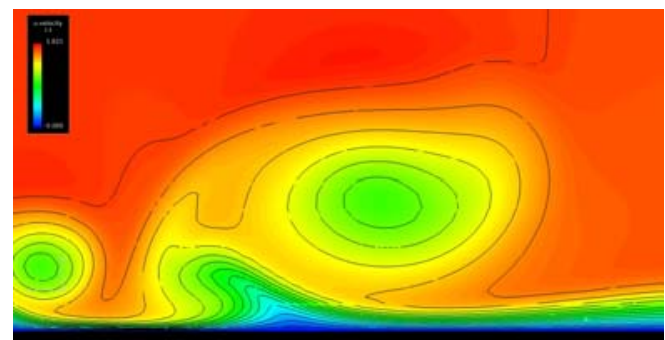

(d) $\beta=35^{\circ}$

Figure 12: Axial velocity fields at different angles of attack measured in a spanwise plane placed five device heights downstream the VG

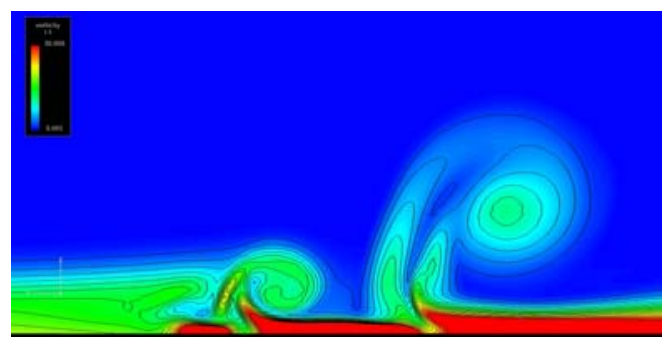

(a) $\beta=20^{\circ}$

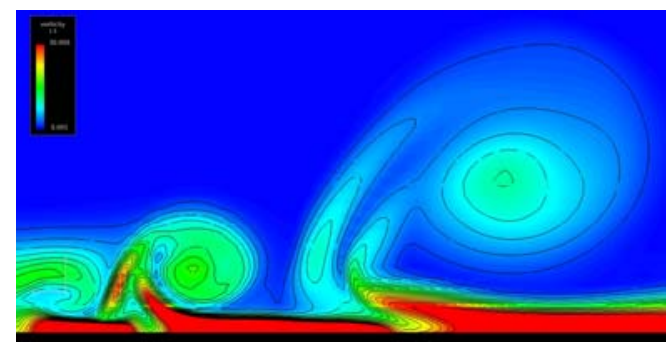

(b) $\beta=25^{\circ}$

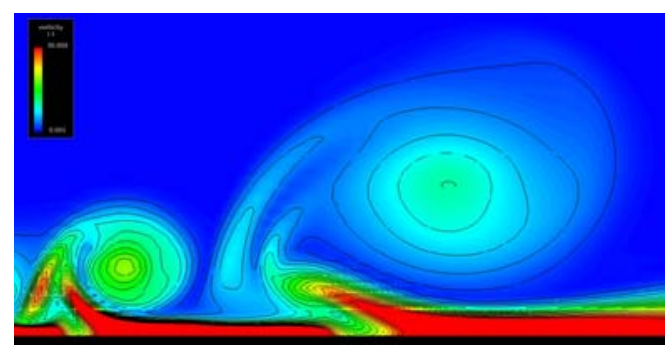

(c) $\beta=30^{\circ}$

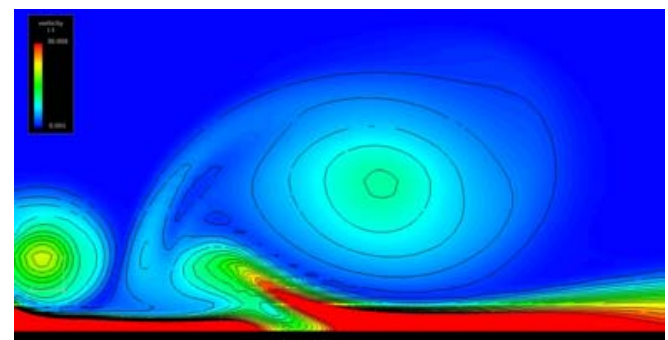

(d) $\beta=35^{\circ}$

Figure 13: Vorticity fields at different angles of attack measured in a spanwise plane placed five device heights downstream the VG 


\section{APPENDIX B}

\section{PRESSURE FIELDS}

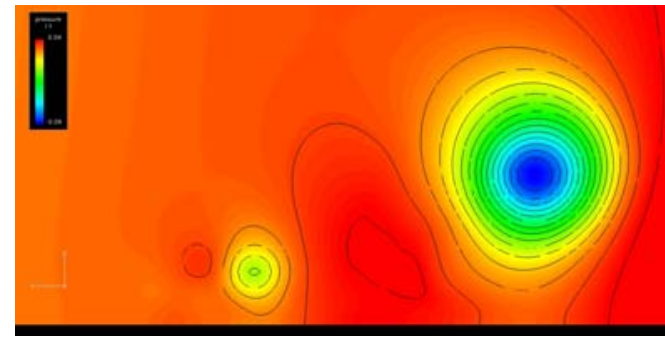

(a) $\beta=20^{\circ}$

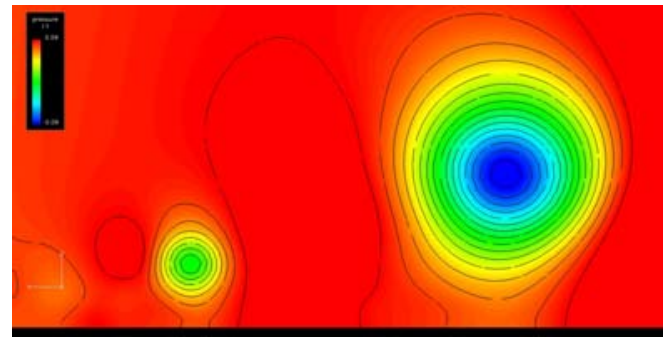

(b) $\beta=25^{\circ}$

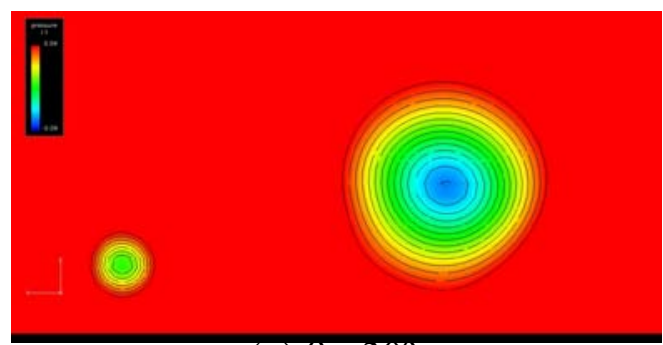

(c) $\beta=30^{\circ}$

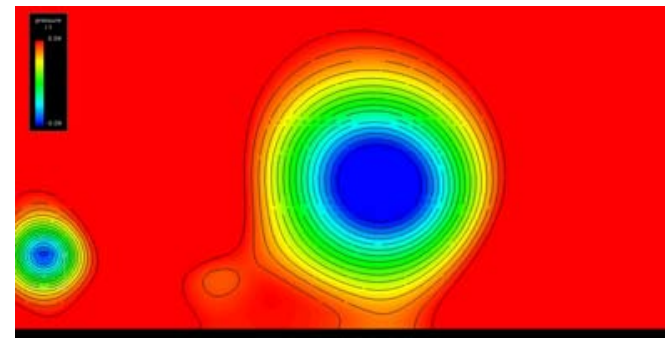

(d) $\beta=35^{\circ}$

Figure 14: CFD pressure fields at different angles of attack measured in a spanwise plane placed five device heights downstream the VG.

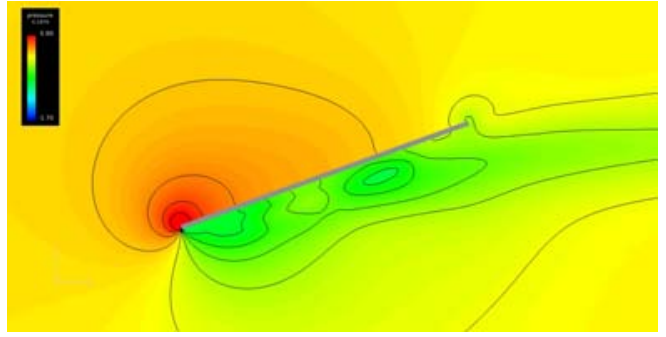

(a) $\beta=20^{\circ}$

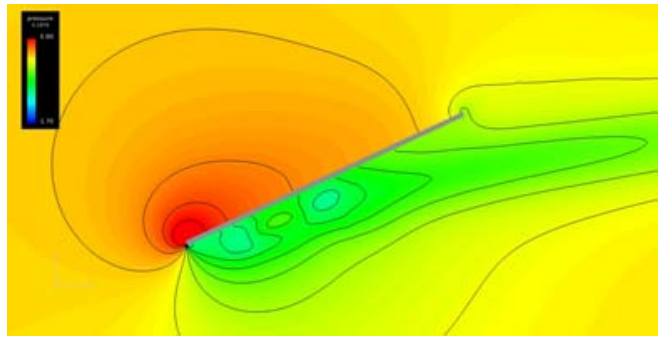

(b) $\beta=25^{\circ}$

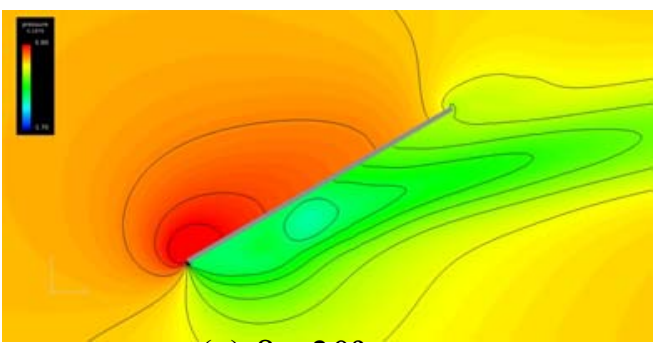

(c) $\beta=30^{\circ}$

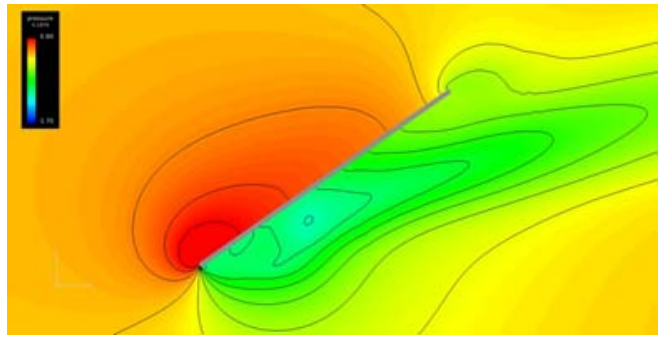

(d) $\beta=35^{\circ}$

Figure 15: Top view CFD pressure fields at different angles of attack measured in a plane parallel to the wall placed 0.8 device heights from the wall. 


\section{APPENDIX C}

AXIAL/AZIMUTAL VELOCITY PROFILES FOR THREE DIFFERENT GRID LEVELS.

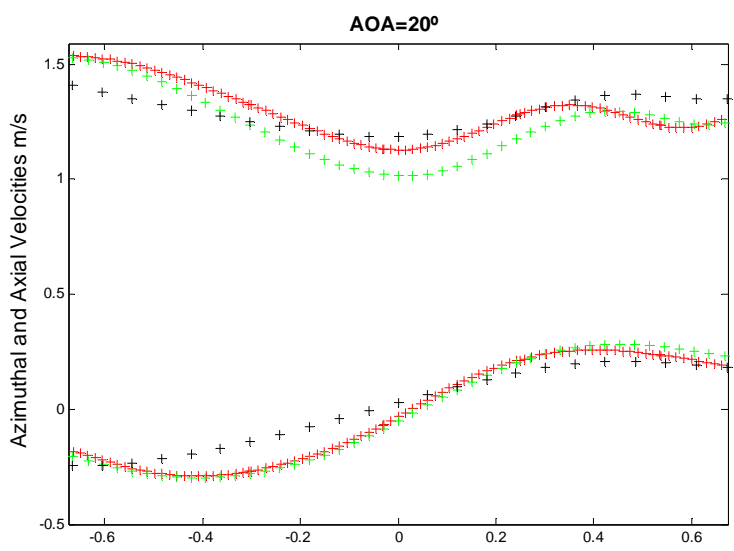

(a) $\beta \stackrel{\text { r/h }}{=} 20^{\circ}$

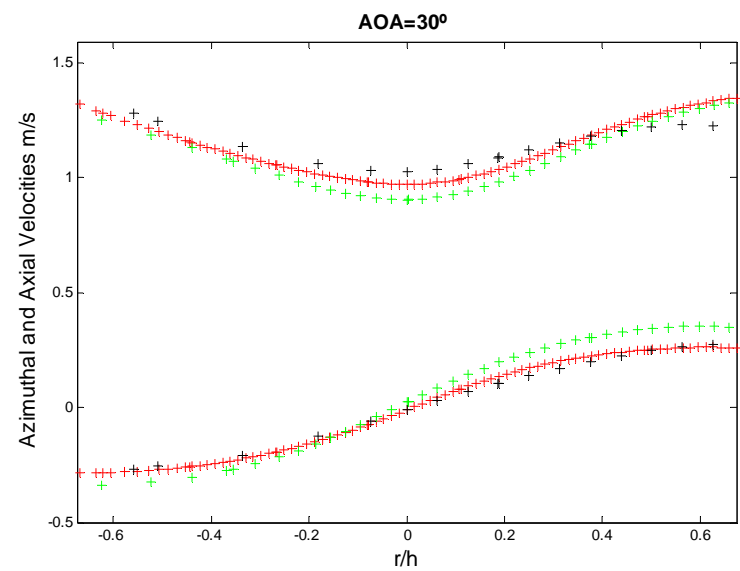

(c) $\beta=30^{\circ}$

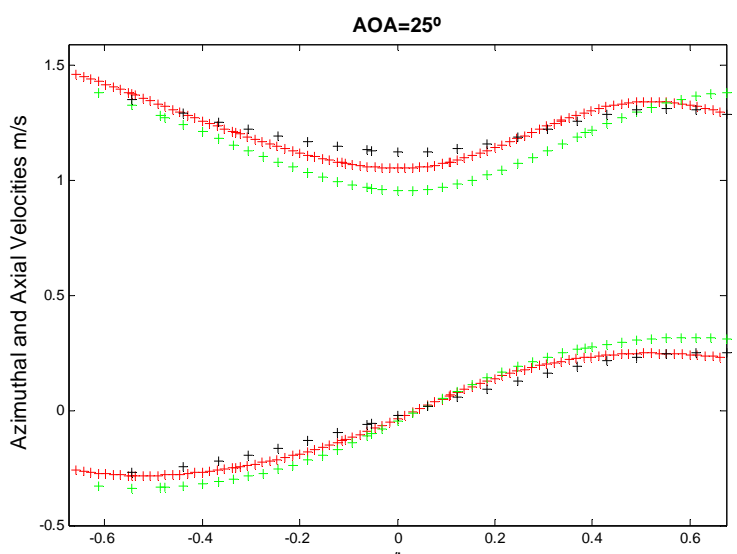

(b) $\beta=25^{\circ}$

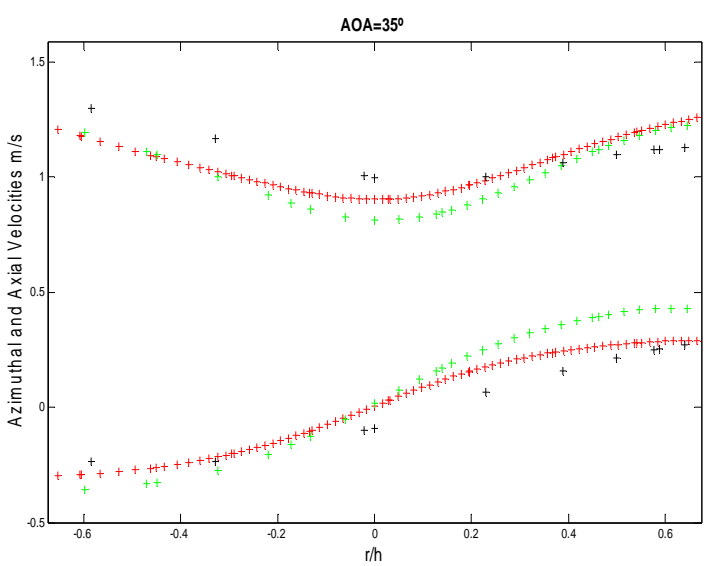

(d) $\beta=35^{\circ}$

Figure 16: Axial (upper) and azimuthal (lower) velocity profiles for three different mesh sizes of the computations. Red colour is the finest mesh, green is the medium mesh size and black is the coarsest one. 